\title{
Neurological Manifestations in Pediatric Inflammatory Multisystem Syndrome Temporally Associated with SARS-CoV-2 (PIMS-TS)
}

\author{
Sagar S. Lad ${ }^{1,2}$ (D) $\cdot$ Santosh Pandurang Kait ${ }^{1} \cdot$ Pradeep B. Suryawanshi $^{1} \cdot$ Jalil Mujawar $^{1} \cdot$ Preeti Lad $^{1}$. \\ Ravindra Khetre ${ }^{1}$ - L. M. Jadhav ${ }^{1} \cdot$ Amol Bhor $^{1}$ • Prasad Balte ${ }^{1}$ - Prateek Kataria ${ }^{1} \cdot$ Mohd Shahbaz Saifi $^{1} \cdot$ Shehla Kazi $^{1}$
}

Received: 20 August 2020 / Accepted: 5 October 2020 / Published online: 20 October 2020

(C) Dr. K C Chaudhuri Foundation 2020

To the Editor: Till recently, it was assumed that pediatric population had relatively benign course in SARS-CoV-2 infection. However, since April 2020, there have been an increased number of reports, regarding Pediatric Inflammatory Multisystem Syndrome Temporally Associated with SARSCoV-2 (PIMS-TS) [1-3]. Only a handful of studies have reported neurological symptoms in PIMS-TS and most of these findings are from adult studies. Neurological manifestations reported include headache, encephalitis, meningitis, encephalopathy, strokes, seizures, and anosmia [4].

In July 2020, four out of five children diagnosed with confirmed PIMS-TS presented to us with neurological symptoms. These included convulsions with encephalopathy in a 7-y-old boy with toxic shock syndrome. Cerebrospinal fluid (CSF) examination was normal but MRI brain showed signal changes in splenium of corpus callosum (SCC). EEG showed diffuse slow activity. The second child was a 13-y-old girl with shock (CSF and MRI was not performed). The third child was a 2-y-old boy who had new onset of convulsions with persistent fever (CSF, EEG and MRI - normal). The fourth child presented as Kawasaki like disease and had excessive sleepiness and lethargy (CSF and MRI - normal).

Similar findings of new-onset neurological symptoms like encephalopathy, headache, brainstem signs with dysarthria or dysphagia, meningism and cerebellar ataxia have been reported by Abdel-Mannan et al. in 14.8\% of PIMS-TS children. MRI findings of their study showed signal changes in SCC

Sagar S. Lad

drsagarlad@yahoo.com

1 Pediatric Intensive Care Unit, Sahyadri Super Speciality Hospital, Nagar Road, Shastrinagar, Pune, Maharashtra 411006, India

2 Jehangir Hospital, 32 Sassoon Road, Pune, Maharashtra 411001, India region in all four cases which was seen in one child in our study. These reversible lesions of SCC are thought to represent focal intramyelin edema secondary to inflammation [1]. Similar neurological findings have been reported in studies by Whittaker et al. and Chiotos et al. [2, 3].

Angiotensin-converting enzyme 2 receptors (ACE2) is expressed in neurons and glial cells and attachment of virus to ACE2 receptor causes injury, leading to direct viral invasion in the central nervous system (CNS). An immunemediated neuronal damage due to cytokine storm was considered as a possible mechanism of CNS manifestations. Other possible mechanism can be exposure of immune system to new CNS antigens as a result of blood-brain barrier damage from SARS-CoV-2, causing endotheliopathy which leads to an immune directed attack on CNS [4, 5].

In conclusion, PIMS-TS should be considered as a differential diagnosis for children presenting with unexplained neurologic symptoms with or without systemic involvement in current scenario. Close neurodevelopmental surveillance is required to assess the neurological and cognitive outcomes in these children.

\section{Compliance with Ethical Standards}

Conflict of Interest None.

\section{References}

1. Abdel-Mannan O, Eyre M, Löbel U, et al. Neurologic and radiographic findings associated with COVID-19 infection in children. JAMA Neurol. 2020. https://doi.org/10.1001/jamaneurol.2020. 2687.

2. Whittaker E, Bamford A, Kenny J, et al. Clinical characteristics of 58 children with a pediatric inflammatory multisystem syndrome temporally associated with SARS-CoV-2. JAMA. 2020. https://doi.org/ 10.1001/jama.2020. 
3. Chiotos K, Bassiri H, Behrens EM, et al. Multisystem inflammatory syndrome in children during the coronavirus 2019 pandemic: a case series. J Pediatric Infect Dis Soc. 2020;9:393-8.

4. Aghagoli G, Gallo Marin B, Katchur NJ, Chaves-Sell F, Asaad WF, Murphy SA. Neurological involvement in COVID-19 and potential mechanisms: a review. Neurocrit Care. 2020. https://doi.org/10. 1007/s12028-020-01049-4.
5. Chen T-H. Neurological involvement associated with COVID-19 infection in children. J Neurol Sci. 2020;418:117096.

Publisher's Note Springer Nature remains neutral with regard to jurisdictional claims in published maps and institutional affiliations. 